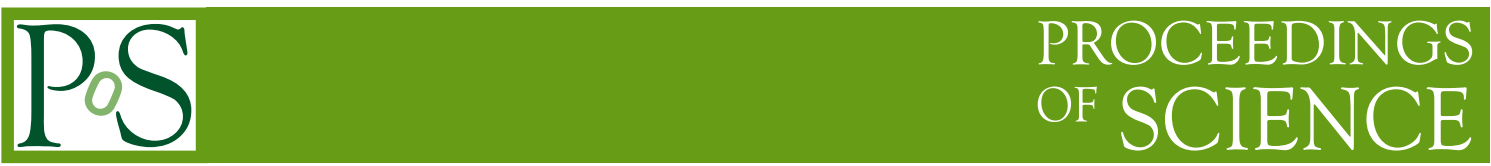

\title{
Gravitational Radiation from Neutron Stars and Black Holes
}

\author{
Carlos F. Sopuerta* \\ Institut de Ciències de l'Espai (CSIC), Facultat de Ciències, Campus UAB, Torre C5 parells, \\ E-08193 Bellaterra, Spain \\ Institut d'Estudis Espacials de Catalunya (IEEC), Ed. Nexus-201, Gran Capità 2-4, E-08034 \\ Barcelona, Spain \\ E-mail: sopuertalieec.uab.es
}

\begin{abstract}
Gravitational Wave Astronomy is becoming a reality as Earth-based interferometric gravitationalwave detectors reach the design sensitivities and move towards advanced configurations that may lead to gravitational-wave detections in the immediate future. In this contribution, I briefly summarize the basic characteristics of this new area, the discovery prospects and the potential for fundamental physics. Then, I present results of some investigations of two different sources of gravitational waves that are potential targets for present and future planned observatories. First, I will discuss the generation of gravitational radiation by non-linear effects arising from the coupling between radial and non-radial oscillations of neutron stars, which may produce distinctive gravitational-wave signatures. The gravitational radiation emitted by these sources is in the frequency band of Earth-based detectors. And second, I will discuss the gravitational-wave emission during the inspiral of extreme-mass-ratio compact binaries. In this case, the gravitational waves have low frequencies, inside the frequency band of space observatories like LISA.
\end{abstract}

Supernovae: lights in the darkness (XXIII Trobades Científiques de la Mediterrània)

October 3-5 2007

Maó, Menorca, Spain

\footnotetext{
* Speaker.
} 


\section{Introduction}

Detection and study of the gravitational radiation coming from astrophysical and cosmological sources is the main task of the emergent area of Gravitational Wave (GW) astronomy. This task is coming closer to reality as Earth-based detectors like LIGO [1] and VIRGO [2] are improving their sensitivities. At the same time, there is a strong international effort to construct a GW observatory in space, the Laser Interferometer Space Antenna (LISA) [3], an ESA-NASA mission that is expected to be launched in the next decade, with an ESA precursor mission, LISA PathFinder [4], that will be launched in 2010 .

Gravitational radiation has physical properties that make it a very attractive tool for astronomy. In particular, the weak coupling of gravity with matter implies that the GWs that our observatories will detect carry nearly uncorrupted information from the sources that produced them. At the same time, this implies that GWs are quite difficult to detect since they will also interact weakly with the detector. In many cases the signal will be buried in the instrumental noise, and in some cases, in GW backgrounds (for instance, LISA will be limited by the GW background produced by galactic stellar compact binaries). Therefore, an important task of GW astronomy is to construct theoretical waveform templates in order to separate in an efficient way the signal from the noise using statistical techniques like match filtering. Depending on the GW source, these templates can be obtained by full numerical relativity simulations, relativistic perturbation theory, or post-Newtonian approximation schemes, or by a combination of them. This is a research area in GW astronomy where a significant part of the theoretical efforts have been directed to. There are other important topics that theory is dealing with that are important for the development of GW astronomy. In particular, the study of the formation mechanisms and rates of GW sources. These studies are relevant for the design of the GW observatories. They can also determine the regions of the configuration space of the potential GW sources that correspond to astrophysically realistic situations. This last point is crucial to establish the parameter space that GW template banks have to cover, and hence to determine the range of initial conditions for the simulations of GW sources. For instance, in the case of black hole $(\mathrm{BH})$ binaries we are interested in the range of orbital parameters and spin orientations that we can expect from each of the possible astrophysical scenarios that yield BH binaries. Finally, another important arena for theoretical studies is the investigation of possible electromagnetic counterparts to GW events (GRBs, X-rays, etc.) and their impact on on GW astronomy, whereby synergies between electromagnetic and GW observations are explored.

The main sources of gravitational radiation for Earth-based interferometric detectors are: stellarmass compact binaries [BH-BH, BH-Neutron Star (NS), and NS-NS binaries], supernovae core collapse, relativistic pulsars, oscillations of neutron stars (w-modes, r-modes, etc.), and stochastic sources (astrophysical and cosmological). On the other hand, the main sources for space-based detectors like LISA are: Galactic compact binaries [e.g. White Dwarf (WD) binaries], massive BH binary mergers, capture of stellar-mass compact objects by massive BHs sitting at galactic centers [known as extreme-mass-ratio inspirals (EMRIs)], and cosmological backgrounds. Remarkable advances in astrophysics, cosmology and gravitational physics are expected from observations of these systems. To illustrate this, let us mention a few outcomes of GW astronomy: Direct proof of the existence of Gravitational Radiation and its properties (we have an indirect proof of their existence through the observations of the binary pulsar PSR B1913 + 16 [5] discovered by Hulse 
and Taylor [6]); information about masses, spins, and orbital parameters for relativistic compact binaries; tests of the no-hair theorem which, roughly speaking, says that astrophysical BHs are characterized only by their mass and intrinsic angular momentum; information about the equation of state of NSs, rotational properties, progenitor masses, etc.; tests of galaxy formation models and understanding of the growth history of massive $\mathrm{BHs}$; study of the expansion history of the universe by combining gravitational and electromagnetic observations of sets of GW standard candles like massive BH binary mergers or EMRI events; etc. As a consequence, it is expected that GW astronomy will open a new window to the exploration of the Universe in a similar way as it happened in the past with the opening of new electromagnetic windows. Moreover, there is a considerable potential for observation of unexpected GW sources, and it is also possible that these sources may provide information relevant not only for astrophysics and cosmology but also for fundamental physics.

An outcome of GW astronomy that deserves a separate comment is the possibility of testing General Relativity and putting constraints on alternative theories of gravity [7]. The first such constraints came from solar system observations, but the gravitational fields involved in the solar system are weak and the velocities are small as compared with the speed of light. Therefore, these observations can only test the non-radiative weak-field limit of relativistic theories of gravity. The situation changed after the discovery of the binary pulsar PSR B1913+16 [6] (which has been followed by other binary pulsars: PSR B1534+12, PSR J1141-6545, PSR J1829+2456, PSR J0737-3039), as this system involves regions with strong gravitational fields (inside and outside the components of the binary). The way in which certain alternative theories of gravity can be constrained by binary pulsar observations is by the timing of phenomenological post-Keplerian parameters (see [8] for a detailed and recent review of tests of gravity theories by using binary pulsars). For a given theory, one can compute these post-Keplerian parameters (eight) in terms of the Keplerian ones (five) and the masses of the binary (two). Therefore, the measurement of a postKeplerian parameter determines a curve in the mass plane (the plane of the possible masses of the binary components). Then, the measurement of three post-Keplerian parameters constitutes a test of the gravity theory under consideration. Such a test is passed if the three curves intersect at one point (if one adds the measurement error bars the thick curves should just have a non-empty intersection). In the cases in which $n(n \geq 3)$ post-Keplerian parameters are measured, $n-2$ independent tests of the gravitational theory can be performed.

However, the tests just mentioned obviously do not involve GW measurements. To illustrate the potential of GW astronomy in this respect, let us mention the constraints that LISA observations can impose on the coupling parameter of scalar-tensor theories of the Brans-Dicke type, $\omega_{B D}$. In [9], assuming a $10^{3} M_{\odot} B H+1 N S$ inspiral at $50 \mathrm{Mpc}$ and 1 year of integration prior the last stable orbit, it was shown that it is possible to get the following bound: $\omega_{B D}>3 \times 10^{5}$ (the Cassini mission [10] yields $\omega_{B D}>4 \times 10^{4}$ ). Moreover, it was also shown that LISA observations can also impose bounds on the Compton wavelength $\lambda_{g}$ of the graviton [9]: Assuming a $10^{6} M_{\odot} \mathrm{BH}$ binary inspiral at $3 \mathrm{Gpc}$ we can get $\lambda_{g}>5.4 \times 10^{16} \mathrm{~km}$ (4 orders of magnitude larger than with solar system dynamics). In [11], these estimations were revised by including the effect of spin couplings. It was found that the bound on the Brans-Dicke parameter is significantly reduced by spin-orbit and spin-spin couplings (factors of 10 to 20), while the bound on the graviton Compton wavelength is only marginally reduced (factors of 4 to 5 ). 
As it has been mentioned in this introduction, NSs and BHs (of different sizes) are among the most important sources of GWs, mainly due to their strong gravity. However, this same property also makes them very challenging for the task of making theoretical predictions of the shape and amplitude of the GWs that they emit. In what follows, I will describe some work on the topic of the description of sources of GWs in which I have participated with other researchers in the recent past. More specifically, I will discuss some results on the description of two very different types of sources of GWs: (i) GWs from non-linear oscillations of relativistic stars, which are relevant for Earth-based GW observatories. (ii) Simulations of EMRI events and the GWs emitted by them, which is of interest for space-based observatories like LISA.

\section{Gravitational Radiation from non-linear oscillations of relativistic stars}

Neutron stars can undergo oscillating phases during their dynamical evolution. Astrophysical scenarios in which these oscillations can be relevant for GW astronomy are: oscillations in a binary systems close to merger due to the tidal force exerted by the companion during the coalescence; oscillations in a proto-neutron star after the core bounce due either to the bounce dynamics or to the fall-back accretion of material, which has not been expelled by the supernova shock; etc (see [12] for a recent review).

Gravitational radiation is one of the dissipative processes that damp the stellar oscillations carrying away important information about the physical properties and structure of the star. These oscillations generate high frequency GWs, above $500-600 \mathrm{~Hz}$, and therefore are targets for Earthbased interferometers like LIGO and VIRGO $[1,2]$. In order to increase the chances of a detection, it is necessary to increase our theoretical understanding of the sources and provide more accurate templates of the GW signals.

Linear perturbation theory is appropriate to describe the dominant features of stellar pulsations, but it neglects important details due to non-linear effects. For instance, we need non-linear perturbation theory in order to understand energy transfer between different oscillatory modes and how this can saturate the $f$-mode and $r$-mode instabilities of rotating stars or limit the persistence of bar-mode instabilities. Other relevant non-linear effects are the development of $g$-mode instabilities in core collapse supernovae, and mass-shedding-induced damping of oscillations in rapidly rotating stars. On the other hand, there is a wealth of literature dedicated to the understanding of the generation of gravitational waves by linear oscillations of relativistic stars (see [13] and references therein). However, we know relatively little about non-linear dynamical effects (mainly due to numerical studies. See, e.g. [14]).

Despite the fact that strong non-linear effects require a fully non-perturbative approach, it is reasonable to expect that some interesting physical phenomena may involve a mild non-linearity for which a second-order perturbative treatment should provide a reasonable description. Along these lines, an interesting scenario to study is the one in which a NS is oscillating radially and non-radially. At first order, radial oscillations of a spherical star don't emit per se any GWs, but they can drive and possibly amplify non-radial oscillations and then produce gravitational radiation to a significant level. For instance, radial and non-radial oscillations are expected to be prevalently excited after a core bounce. Even though the quadrupole component provides the dominant contribution to the gravitational radiation, the radial pulsations may store a considerable amount of 
kinetic energy and transfer a part of it to the non-radial perturbations. As a result, this non-linear interaction could produce a damping of the radial pulsations and hence, an interesting GW signal. The strength of this signal depends naturally on the efficiency of the coupling, which is the effect we want to explore. In addition, one may expect the appearance of non-linear harmonics, which may also come out at lower frequencies than the linear modes [14], where the Earth-based laser interferometers have a higher sensitivity.

Following this motivation, we started investigating whether non-radial oscillations can be driven or even amplified through coupling by an internal radial oscillation, regardless of the presence of an external source. These non-linear processes can occur, for instance, in a proto-neutron star that is still pulsating. A mainly radial pulsation could, for example, drive the non-radial oscillations, either naturally present, or excited through fall-back accretion.

To carry out this study we employed a non-linear multi-parameter perturbative formalism [15, 16] in order to develop a gauge-invariant perturbative scheme for studying, in the time domain, the coupling between the radial pulsations and, both polar [17] and axial [18], non-radial oscillations. The time-domain numerical simulations in the axial case were done in [18], whereas in the polar case were done in [19]. For simplicity, here we will focus on the axial case.

As it was shown in $[17,18]$, the structure of the perturbative equations is hierarchical. That is, first we need to introduce a background spacetime that will represent the situation corresponding to no oscillations, that is, a stationary star (equilibrium configuration). Then, we can compute the first-order oscillations (radial and non-radial) of the stationary star, and finally, using all these ingredients, we can compute the perturbations describing the non-linear coupling between linear modes.

The equilibrium configuration (the background spacetime) is a perfect-fluid spherically symmetric relativistic star. Such a model is determined by the solution of the Tolman-OppenheimerVolkoff equations given an equation of state. In our case, we assumed a polytropic equation of state: $p=K \rho^{\Gamma}$ with adiabatic index $\Gamma=2$ and $k=100 \mathrm{~km}^{2}$. Prescribing a central mass energy density $\rho_{c}=3 \times 10^{15} \mathrm{~g} \mathrm{~cm}^{-3}$, one obtains the following values for the stellar mass and radius: $M=1.26 M_{\odot}$ and $R=8.862 \mathrm{~km}$.

Then, we have two classes of linear oscillations: (i) the radial pulsations, which correspond to the $\ell=0$ mode, and (ii) the axial non-radial oscillations with harmonic number $\ell \geq 2$. The radial perturbations are completely described by a set of four perturbative variables, two metric perturbations and two fluid variables, which obey three first-order evolution equations and two constraints (reflecting the fact that there is just one single radial degree of freedom). We can set up a hyperbolic-elliptic problem where the Hamiltonian constraint is solved at any time step for one of the metric variables. The axial non-radial perturbations can be described by a system of two equations, the axial wave master equation and a conservation equation. The former governs the evolution of the only gauge invariant metric variable of the axial sector, let us call it $\Psi^{N R}$, while the latter is an equation for the axial velocity perturbation, $\beta^{N R}$. At first order, the stationary character of the axial velocity allowed us to study separately the spacetime dynamical degree of freedom and its stationary part. In particular, the stationary solution describes the differential rotation induced on the background star by the $\ell$-mode of the velocity perturbation, and the associated metric perturbation describes the dragging of inertial frames.

The axial non-linear coupling perturbations can be again described by a gauge invariant metric 
perturbation, let us call it $\Psi^{C}$, and an axial velocity perturbation, $\beta^{C}$. In this case, in the stellar interior, these non-linear perturbations satisfy inhomogeneous linear equations. The homogeneous part of these linear equations is constructed exactly from the same linear operators as the equations for the first-order axial non-radial perturbations. The inhomogeneous part, the source term, is the sum of products of first-order radial perturbations times first-order axial non-radial perturbations. In the exterior we do not have matter fields, and hence the dynamics is described again by ReggeWheeler-type master equations. Stellar interior and exterior communicate through the general relativistic junction conditions at the surface of the star.

We have evolved these perturbative equations for two types of initial configurations: (i) a differentially rotating and radially pulsating star, and (ii) the scattering of a gravitational wave by a radially pulsating star. We will focus on the former configuration, as the dynamics of coupling oscillations is more interesting (for a complete description see [20]).

The initial configuration for the radial pulsations has been excited by selecting specific radial eigenmodes. We have chosen an origin of time such that the radial eigenmodes are described only by the eigenfunctions associated with the radial velocity perturbation $\gamma^{R}$ (see [18]). The eigenfunctions and eigenvectors are found by solving the Sturm-Liouville problem associated with this perturbative variable. Using a numerical code based on the relaxation method we were able to determine the eigenfrequencies of the radial modes with an accuracy better than 0.2 percent, as compared with values in the literature. It has been checked that the simulations done with an arbitrary initial radial mode satisfy, with high accuracy, the Hamiltonian constraint and are stable for very long evolutions. The radial spectrum, which has been determined by applying a fast Fourier transformation to the time evolution, reproduce the results in the literature with an accuracy better than 0.2 percent.

The perturbations describing the axial differential rotation are obtained by expanding in vector harmonics the relativistic version of the $j$-constant rotation law and taking the first component, the one that is related to the GW emission, that is, the $\ell=3$ mode. The initial profile of the axial velocity perturbations contains two specifiable parameters: a parameter $A$ that controls the amount of differential rotation, and the angular velocity at the rotation axis $\Omega_{c}$. The value for $A$ has been chosen in order to have a smooth profile and a relatively high degree of differential rotation, since for high $A$ the rotation tends to be uniform and then the $\ell=3$ mode vanishes. On the other hand, we have chosen an angular velocity that corresponds to a $10 \mathrm{~ms}$ rotation period at the axis of the star. Once we have solved the perturbative equations and obtained the GW signal for this particular angular velocity, we can make use of the linear character of the perturbative equations to obtain the same result for any other angular velocity just by applying the corresponding rescaling. Finally, in order to study the effects of the coupling between the linear oscillations we have taken zero initial data for the coupling perturbations.

Numerical simulations of a radially pulsating and differentially rotating star have shown a new interesting gravitational signal. The gravitational waveforms exhibit the following properties: (i) An excitation of the first $w$-mode at the beginning of the evolution. (ii) A periodic signal driven by the radial pulsations present in the source term of the coupling perturbations (see Fig. 1). This picture is confirmed by the computation of spectrum of the perturbations, where we have noticed that the radial normal modes are precisely mirrored in the GW signal corresponding to the nonlinear perturbations. On the other hand, the excitation of the $w$-mode at the early stages of the 

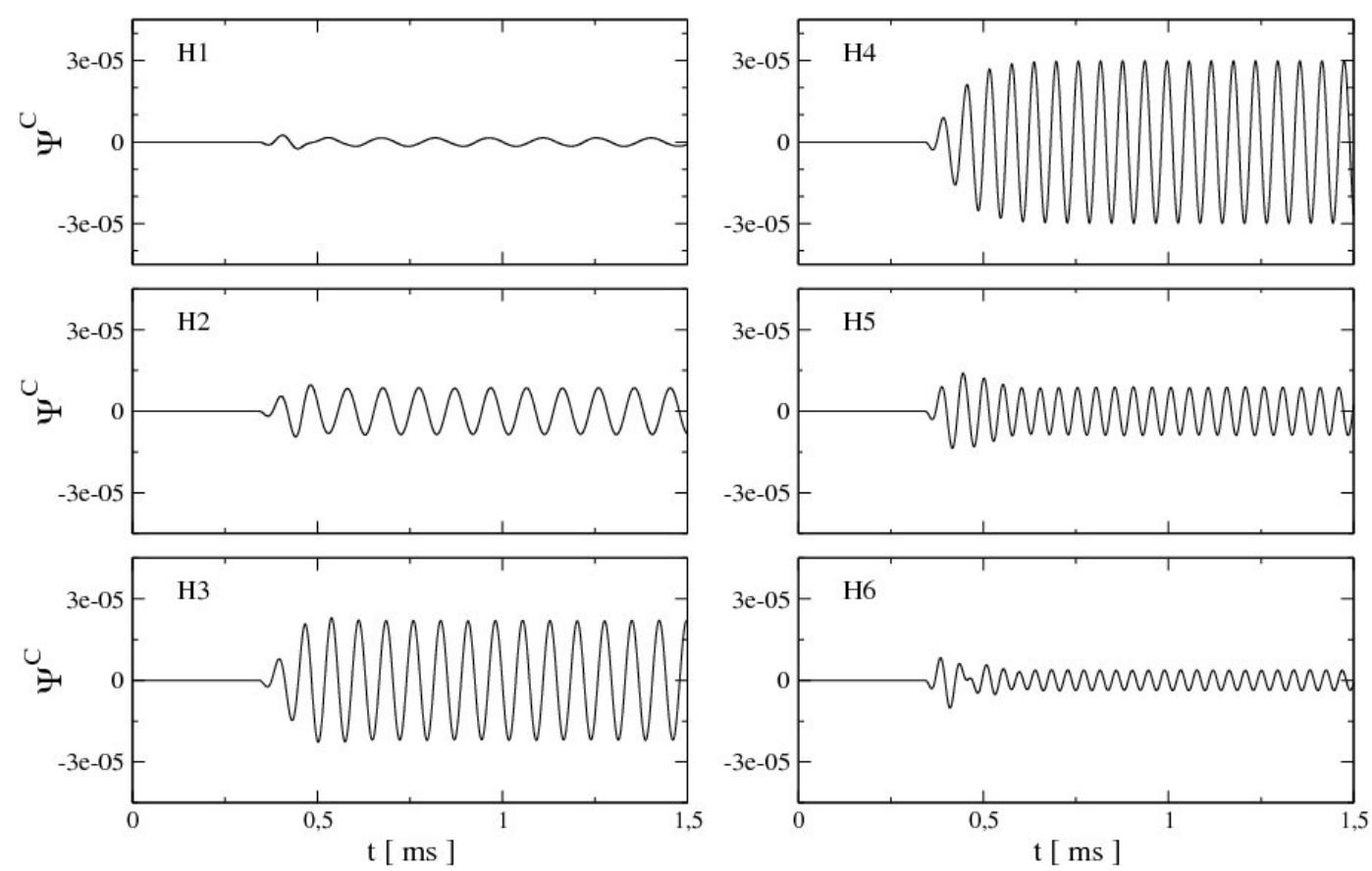

Figure 1: Comparison of six $\Psi^{C}$ waveforms, in $\mathrm{km}$, for the $\ell=3$ multipole. The radial pulsations considered correspond to single mode oscillation from $\mathrm{H} 1$ to $\mathrm{H} 6$ overtone. These plots show that a resonance effect take place in $\Psi^{C}$. See text for a discussion.

numerical simulations is an unphysical response of the system to small initial violations of the axial constraint equations for the coupling perturbations.

In Fig. 1, we can see that the metric waveforms associated with the coupling perturbations, and described by the axial master function $\Psi^{C}$, exhibit an interesting amplification when the radial oscillations pulsate at frequencies close to the $\ell=3$ axial spacetime $w$-mode, $v_{w}=16.092 \mathrm{kHz}$. For the particular stellar model under consideration this effect appears at the third and fourth radial overtones, whose frequencies are $13.545 \mathrm{kHz}$ and $16.706 \mathrm{kHz}$ respectively. It is worth mentioning that this effect takes place despite the fact that the energy and the maximum displacement of the surface of the radial modes decrease proportionally to the order of the radial modes (see Table 1). We can interpret this amplification as a resonance between the radial frequencies, the source terms in the coupling perturbative equations behave as forcing terms, and the natural frequencies of the axial oscillations, which satisfy a wave-like master equation.

An important remark is that our perturbative approach does not include backreaction effects. That is, it cannot account for the damping of the radial oscillations or the slowing down of the stellar rotation due to the contribution of the non-linear coupling to the energy loss in GWs. Backreaction effects can be studied by looking at higher perturbative orders. Nevertheless, we can provide a rough estimate of the damping time of the radial pulsations by assuming that the energy emitted is completely supplied by the first-order radial oscillations, and that the power radiated in GWs is constant in time. In this way, the damping time is given by the following expression:

$$
\tau_{\ell m}^{C} \equiv \frac{E_{n}^{R}}{<\dot{E}_{\ell m}^{C}>}
$$




\begin{tabular}{|c|c|c|c|c|c|c|}
\hline $\begin{array}{c}\text { Radial } \\
\text { Mode }\end{array}$ & $\begin{array}{c}\text { Frequency } \\
{[\mathrm{kHz}]}\end{array}$ & $\begin{array}{c}E_{n}^{R} \\
{\left[10^{-8} \mathrm{~km}\right]}\end{array}$ & $\begin{array}{c}\xi_{\mathrm{sf}}^{R} \\
{[\mathrm{~m}]}\end{array}$ & $\begin{array}{c}<\dot{E}_{30}^{C}> \\
{\left[10^{-14}\right]}\end{array}$ & $\begin{array}{c}\tau_{30}^{C} \\
{[\mathrm{~ms}]}\end{array}$ & $N_{\text {osc }}$ \\
\hline $\mathrm{F}$ & 2.138 & 35.9 & 12.65 & $1.54 \times 10^{-6}$ & $7.78 \times 10^{10}$ & $1.67 \times 10^{11}$ \\
$\mathrm{H} 1$ & 6.862 & 4.2 & 4.02 & $5.69 \times 10^{-2}$ & $24.59 \times 10^{4}$ & $1.69 \times 10^{6}$ \\
$\mathrm{H} 2$ & 10.302 & 1.37 & 2.66 & 4.04 & $11.29 \times 10^{2}$ & $1.16 \times 10^{4}$ \\
$\mathrm{H} 3$ & 13.545 & 0.62 & 2.02 & 46.69 & 44.28 & $5.99 \times 10^{2}$ \\
$\mathrm{H} 4$ & 16.706 & 0.34 & 1.64 & 130.84 & 8.64 & $1.44 \times 10^{2}$ \\
$\mathrm{H} 5$ & 19.823 & 0.21 & 1.38 & 15.90 & 44.04 & $8.73 \times 10^{2}$ \\
$\mathrm{H} 6$ & 22.914 & 0.14 & 1.19 & 3.71 & 126.12 & $2.89 \times 10^{3}$ \\
\hline
\end{tabular}

Table 1: Quantities associated with radial normal modes and their coupling to the first-order axial differential rotation: Energy, $E_{n}^{R}$, and maximum stellar surface displacement $\xi_{\mathrm{sf}}^{R}$ of the radial eigenmodes for initial conditions with velocity amplitude 0.001 ; average power, $\left\langle\dot{E}_{30}^{C}\right\rangle$, emitted in gravitational waves to infinity from the coupling between the radial eigenmode and the axial differential rotation; estimated values of the damping times, $\tau_{30}^{C}$; and number of oscillation periods, $N_{\mathrm{Osc}}$, that takes for the non-linear oscillations to radiate the total energy initially contained in the radial modes.

where $E_{n}^{R}$ is the energy of a radial eigenmode (see Table 1), and $\left\langle\dot{E}_{l m}^{C}\right\rangle$ is the averaged value of the non-linear coupling contribution to the power emitted. The results for $\tau_{30}^{C}$ are shown in Table 1 . In addition, in the last row of Table 1 we have given an estimation of the damping of the radial pulsations associated with a certain radial eigenmode in terms of the number of oscillation cycles:

$$
N_{\mathrm{osc}}=\frac{\tau_{\ell m}^{C}}{T_{n}}
$$

where $T_{n}=v_{n}^{-1}$, with $v_{n}$ being the eigenfrequency of the radial eigenmode. It is interesting to mention that the number of oscillations required for the damping of the $\mathrm{H} 4$ mode is only 12, and hence it would already affect the H4-waveform shown in Figure 1. This is not surprising, and shows that the coupling near resonances is a very effective mechanism for extracting energy from the radial oscillations.

In [19], a similar analysis has been carried out for the case of polar non-radial oscillations, which have a richer spectrum than the axial ones. For typical values of mode-energies expected in the post-bounce phase of core-collapse supernovae it has been found that some bilinear combinations of frequencies may become detectable by the Advanced LIGO and VIRGO detectors [1,2]. Detection of these non-linear modes, together with the information coming from the expected linear modes, would provide new constraints on the possible equations of state, as they also contain information on the radial modes of the star.

To conclude we would like to remark that these studies of non-linear oscillations of relativistic stars indicate the possibility of new interesting features in the GW signal emitted by these systems. To explore further these ideas, additional work should be done on the comparison with full nonlinear simulations and also on improving the stellar model by including all the necessary physical ingredients of astrophysically realistic stars. 


\section{Gravitational Radiation from Extreme-Mass-Ratio Binaries}

Extreme-mass-ratio binaries in the inspiral phase of their evolution (EMRIs), i.e. when the system evolves driven by GW emission, are considered to be a primary source of gravitational radiation to be detected by LISA [3]. They consist of a stellar-mass compact object (SCO), such a main sequence star, a stellar-mass $\mathrm{BH}$, or a NS (with masses in the range $1-10^{2} M_{\odot}$ orbiting a massive $\mathrm{BH}$ (with masses in the range $10^{5}-10^{7} M_{\odot}$ ), hence the mass ratios of interest lie in the range $10^{-3}-10^{-7}$. Their study is expected to provide crucial information about the growth history of galactic massive BHs, tests of the no-hair theorem for BHs, tests of the validity of general relativity, possibility of discerning among different theories of galaxy formation, etc.

Several astrophysical mechanisms that can lead to EMRI events have been proposed (for details, see the review [21]). The most studied is the one known as single captures, in which a SCO in the cusp of the massive BH host galaxy is sent, due to 2-body or multi-body encounters, to an almost radial orbit towards the massive $\mathrm{BH}\left(1-e \sim 10^{-3}-10^{-6}\right)$. As the SCO approaches the massive $\mathrm{BH}$, it loses energy and angular momentum in GWs so that it gets bounded to the massive $\mathrm{BH}$. The resulting orbit shrinks due to the GW emission and when the system enters the LISA frequency band it still has a significantly high eccentricity $(e \sim 0.5-0.9)$. The number of cycles during the last year before plunge is of the order of $10^{5}$ cycles. It has been estimated $[22,23]$ that LISA may detect up to $10^{3}$ EMRI capture events per year. In the last stages of the inspiral, they SCO probes the strong-field region of the central massive $\mathrm{BH}$ and reaches velocities very close to the speed of light. To illustrate this fact we present in Fig. 2 a trajectory that shows the so-called zoom-whirl behaviour, characterized by strong precessional effects, and the associated waveform, which gives an idea of the complex structure of EMRI waveforms. Moreover, these EMRI events constitute precision tools for GW astronomy since the GWs emitted carry a map of the massive $\mathrm{BH}$ spacetime (the massive $\mathrm{BH}$ multipole moments) and it is expected that LISA will be able to measure 3-5 moments with high precision [24-26]. Moreover, it has been predicted [26] that the error in the estimations of mass, spin, and sky location from LISA observations are of the following order: $\Delta\left(\ln M_{\bullet}\right), \Delta\left(\ln m / M_{\bullet}\right), \Delta\left(S_{\bullet} / M_{\bullet}^{2}\right) \sim 10^{-4}, \Delta \Omega \sim 10^{-3}$.

Other EMRI production mechanisms apart from single captures are: (i) Disruption of a stellarmass compact binary passing near a massive BH [27]. (ii) Capture of cores of giant stars close to the massive BH by tidal stresses [28]. (iii) Inspiral of BHs produced in an accretion disc around the massive $\mathrm{BH}[29]$ (at distances $\sim 0.1-1 p c$ ). The EMRIs produced by this mechanism would have equatorial co-rotating circular orbits. Actually, the different EMRI mechanisms mentioned here will produce distinctive GW signals that may allow LISA to distinguish between them. Apart from these astrophysical events, it is interesting to consider two additional systems that are closely related to EMRIs in the sense that can be described using essentially the same techniques. These systems are: (iv) Inspirals of intermediate-mass BHs, with masses in the range $10^{2}-10^{4} M_{\odot}$, located in globular clusters near the central massive BH [30]. (v) Extreme-Mass-Ratio Bursts: long-period, nearly-radial orbits of compact objects around a central massive $\mathrm{BH}$. These are quite similar to EMRI captures, the difference is that the compact object does not get captured, and for this reason the GW emission consists of a few GW bursts produced in a few passages close to the massive BH. Therefore, only systems of galactic origin may be relevant for LISA [31].

Since the GW signal from EMRI events will be buried in the LISA noise, it is crucial to have a 

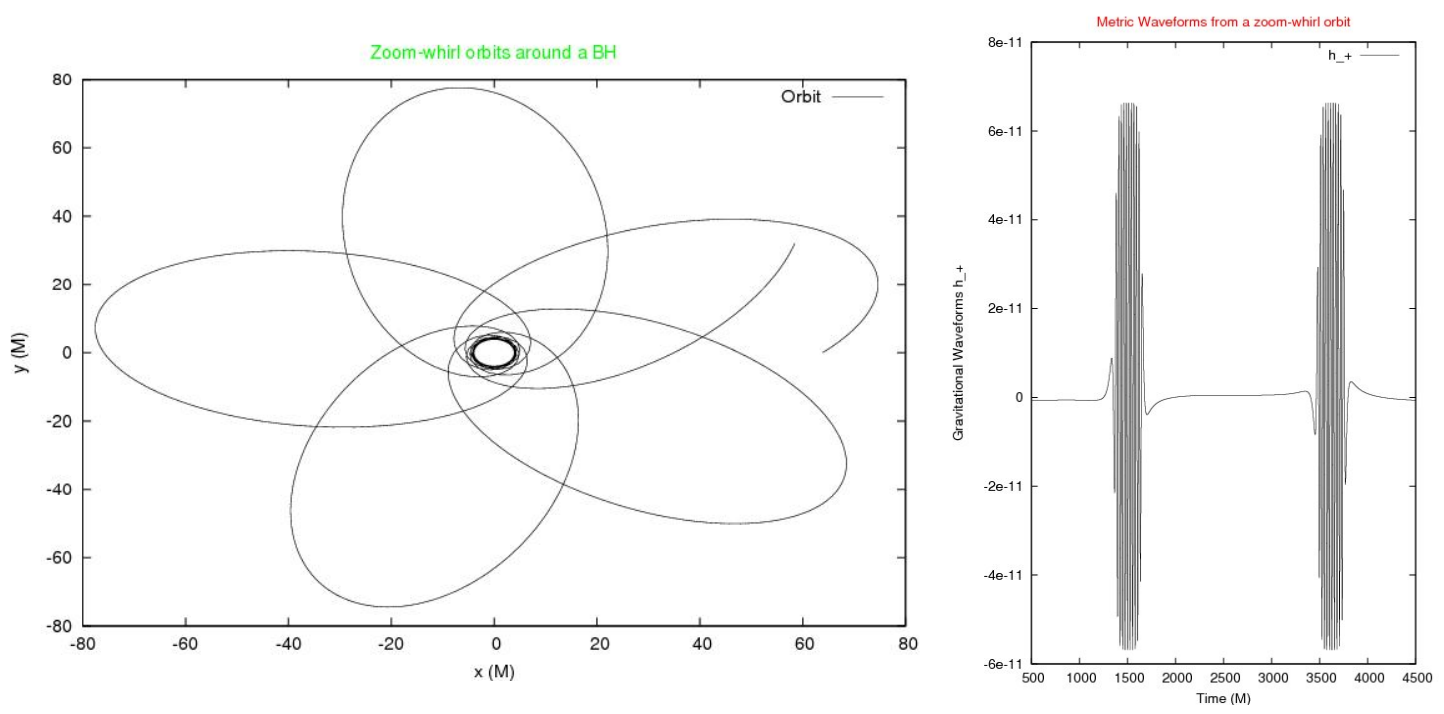

Figure 2: On the left: Zoom-whirl geodesic trajectory of a test mass around a non-rotating BH. The orbit can be parameterized as $r=p M /(1+e \cos \chi)$, where $M$ is the BH mass, $p=7.8001$ is the semilatus rectum, and $e=0.9$ is the eccentricity. On the right: Gravitational waveform (plus polarization) associated with a portion of the zoom-whirl orbit.

good theoretical understanding of their evolution in order to produce accurate waveform templates to be used in data analysis schemes for detection and extraction of physical information from the LISA data stream. Actually, it has been estimated that the theoretical waveform templates must have a precision in the phase better than 1 radian over 1 week for detection, and better than 1 radian over 1 year for extraction of physical parameters.

Due to the small mass ratios involved, BH perturbation theory is an adequate tool for dealing with this problem. The Kerr metric, describing a massive spinning BH, will be the background spacetime to be used in the perturbative scheme, and it will obviously describe the central massive $\mathrm{BH}$ in the absence of other sources of gravitational field. The SCO can then be seen as a matter distribution that induces small spacetime perturbations. In order to compute these perturbations, one usually introduces the additional approximation of treating the SCO as a point-like object. This approximation is justified again by the small mass ratio, but also by the fact that the internal structure of a compact object in General Relativity is effaced to a very high degree of approximation as post-Newtonian calculations have shown. More specifically, it has been estimated that the internal structure of a compact object contributes to order 5PN to the problem of motion.

A key ingredient for the accurate description of the motion of EMRIs is the treatment of the backreaction of the perturbations, that is, how to evaluate the effect of the perturbations generated by the SCO on its own trajectory. The equations of motion for the SCO including backreaction were consistently established in the works [32], in the so-called self-force approach. In this approach, the deviations from geodesic motion (around the BH background spacetime) of the SCO are described in terms of the action of a local force. The practical implementation of the solution of the equations of motion together with the determination of the gravitational waveform is a difficult task that nowadays constitutes an active subject of research by various groups around the world. In part due to this fact, several approximations to the description of EMRIs have been proposed. In 
particular: (i) The adiabatic approximation. It is based on the idea that the long-term evolution may be approximated by the dissipative part of the gravitational self-force [33]. (ii) Klugde waveforms. The idea here is to use approximations that can allow a quick generation of waveform templates (in part for purposes of developing efficient data analysis schemes), which in practice means to find approximations that avoid solving partial differential equations [34].

These approximations may provide useful templates for LISA detections of EMRI events but it seems unlikely that they can provide templates for physical parameter estimations. The key question in this respect is to what extend one can use adiabatic-type approximations, or in other words, how important is the conservative part of the self-force. This is an issue that is presently under debate (see [35]). In any case, self-force calculations can provide a method to compute precise gravitational waveforms for EMRI events, and at the same time, these calculations can be used to test the validity of the adiabatic approximation.

Broadly speaking, the study of the dynamics of EMRIs via the self-force approach involves a number of challenges [36] and can be divided into the following three stages: (A) The computation of the gravitational perturbations produced by the $\mathrm{SCO}$ in the background spacetime of the massive $\mathrm{BH}$, in particular at the SCO location. (B) Solving the equations of motion for the SCO including its own gravitational field [computed in point (A)]. (C) Extraction of the relevant physical information, in particular the gravitational waveforms. None of these parts has been completely solved yet although there has been a number of important advances in the last years. Of particular relevance for the completion of this programme is the computation of the perturbations and the evaluation of the self-force at the SCO location. By one hand, this requires numerical calculations since the equations involved cannot be completely solved analytically and, on the other hand, the physical requirements on the precision of the calculations are quite high. Therefore, it seems that we need to have good enough numerical schemes to perform these calculations. In the last years, timedomain methods have become more and more popular. One of the main reasons is that they can produce accurate results for both very eccentric and almost circular orbits. In contrast, frequencydomain methods have the disadvantage that their convergence becomes very slow as we increase the eccentricity of the orbit.

Numerical techniques in the time domain for self-force calculations is an active area of research. Many of the developments are discussed in the annual CAPRA Ranch meetings on radiation reaction. The present author and collaborators have been investigating the possibility of using Finite Element methods for this task. First, by studying a toy model in scalar gravity that contains all the ingredients (excepting for the spin of the massive BH) of astrophysical EMRI events [37]. The main conclusion of this numerical experiment was that the use of adaptive techniques for the description of EMRIs can significantly improve the efficiency and accuracy of the numerical computations. Second, Finite Element methods have also been used to compute the perturbations generated by a particle orbiting a non-rotating BH in the Regge-Wheeler gauge [38]. In this work, it was shown how Finite Element methods can produce discretizations of the Dirac delta distributions (and their derivatives) present in the perturbative master equations and yield very accurate results for computations of GW fluxes of energy and angular momentum for all type of orbits. Future work will concentrate to extend this work to the case of computations in the Lorenz gauge, a gauge appropriate for self-force calculations, and also to the case of rotating BHs. 


\section{Acknowledgments}

The author acknowledges support from the Ramón y Cajal Programme of the Ministry of Education and Science of Spain and by a Marie Curie International Reintegration Grant (MIRGCT-2007-205005/PHY) within the 7th European Community Framework Programme.

\section{References}

[1] LIGO, http://www.ligo.caltech.edu, http://space.mit.edu/LIGO

[2] VIRGO, http://www.virgo.infn.it

[3] LISA, http://lisa.esa.int, http://lisa.nasa.gov

[4] LISA PathFinder, http://www.esa.int/science/lisapathfinder

[5] J.M. Weisberg and J.H. Taylor, Relativistic binary pulsar B1913+16: Thirty years of observations and analysis, to appear in the proceedings of the Aspen Winter Conference on Astrophysics: Binary Radio Pulsars [astro-ph/0407149].

[6] R.A. Hulse and J.H. Taylor, Discovery of a pulsar in a binary system, ApJ 195 L51 (1975).

[7] C.M. Will, The confrontation between general relativity and experiment, Living Rev. Rel. 44 (2005) [gr-qc/0510072].

[8] T. Damour, Binary systems as test-beds of gravity theories, to appear in the Proceedings of the 6th SIGRAV School in Contemporary Relativity and Gravitational Physics: A Century from Einstein Relativity: Probing Gravity Theories in Binary Systems [arXiv: 0704.074 vv1].

[9] C.M. Will and N. Yunes, Testing alternative theories of gravity using LISA, Class. Quant. Grav. 21 4367 (2004) [gr-qc/ 0403100$].$

[10] B. Bertotti, L. Iess, and P. Tortora, A test of general relativity using radio links with the Cassini spacecraft, Nature 425374 (2003).

[11] E. Berti, A. Buonanno, and C.M. Will, Estimating spinning binary parameters and testing alternative theories of gravity with LISA, Phys. Rev. D 71084025 (2005) [gr-qc/ 041112 9].

[12] K.D. Kokkotas and N. Stergioulas, Gravitational Waves from Compact Sources, in Proceedings of the 5th International Workshop "New Worlds in Astroparticle Physics" [gr-qc/0 506083$].$

[13] N. Andersson, Gravitational waves from instabilities in relativistic stars, Class. Quant. Grav. 20 R105 (2003) [astro-ph/ 0211057$].$

[14] H. Dimmelmeier, N. Stergioulas, J.A. Font, Non-linear axisymmetric pulsations of rotating relativistic stars in the conformal flatness approximation, MNRAS 3681609 (2006) [astro-ph/ 0511394 ].

[15] M. Bruni, L. Gualtieri, and C.F. Sopuerta, Two-parameter nonlinear space-time perturbations: Gauge transformations and gauge invariance, Class. Quant. Grav. 20535 (2003) [gr-qc/0207105].

[16] C.F. Sopuerta, M. Bruni, and L. Gualtieri, Non-Linear N-parameter spacetime perturbations: Gauge transformations through the Baker-Campbell-Hausdorff formula, Phys. Rev. D 70064002 (2004) [gr-qc/0306027].

[17] A. Passamonti, M. Bruni, L. Gualtieri, and C.F. Sopuerta, Coupling of radial and non-radial oscillations of relativistic stars: gauge-invariant formalism, Phys. Rev. D 71024022 (2005) [gr-qc/0407108]. 
[18] A. Passamonti, M. Bruni, L. Gualtieri, A. Nagar, and C.F. Sopuerta, Coupling of radial and axial non-radial oscillations of compact stars: Gravitational waves from first-order differential rotation, Phys. Rev. D 73084010 (2006) [gr-qc/ 0601001 ].

[19] A. Passamonti, N. Stergioulas, and A. Nagar, Gravitational waves from nonlinear couplings of radial and polar nonradial modes in relativistic stars, Phys. Rev. D 75084038 (2007) [gr-qc/ 0702099 ].

[20] A. Passamonti, Non-linear oscillations of compact stars and gravitational waves, PhD Thesis, University of Portsmouth (2005) [gr-qc/ 0607143 ].

[21] P. Amaro-Seoane et al, Astrophysics, detection and science applications of intermediate- and extreme mass-ratio inspirals, Class. Quantum Grav. 24 R113 (2007) [astro-ph/ 07034 95].

[22] J.R. Gair et al, Event rate estimates for LISA extreme mass ratio capture sources, Class. Quant. Grav. 21 S1595 (2004) [gr-qc/ 0405137 ].

[23] C. Hopman and T. Alexander, The Effect of Mass Segregation on Gravitational Wave Sources near Massive Black Holes, ApJ 645 L133 (2006) [astro-ph/ 060332 4].

[24] F.D. Ryan, Gravitational waves from the inspiral of a compact object into a massive, axisymmetric body with arbitrary multipole moments, Phys. Rev. D 525707 (1995); Accuracy of estimating the multipole moments of a massive body from the gravitational waves of a binary inspiral, Phys. Rev. D 561845 (1997).

[25] E. Poisson, Measuring black-hole parameters and testing general relativity using gravitational-wave data from space-based interferometers, Phys. Rev. D 545939 (1996) [gr-qc/9606024].

[26] L. Barack and C. Cutler, LISA capture sources: Approximate waveforms, signal-to-noise ratios, and parameter estimation accuracy, Phys. Rev. D 69082005 (2004) [gr-qc/ 0310125 ].

[27] M.C. Miller, M. Freitag, D. Hamilton, and V.M. Lauburg, Binary encounters with supermassive black holes: Zero-eccentricity LISA events, ApJ 631 L117 (2005) [astro-ph / 0507133 ].

[28] M.B. Davies and A.R. King, The stars of the galactic center, ApJ 624 L25 (2005) [astro-ph/ 0503441 ]; R. Di Stefano, J. Greiner, S. Murray, and M. García, A new way to detect massive black holes in galaxies: The stellar remnants of tidal disruption, ApJ 551 L37 (2001) [astro-ph/0112434].

[29] Y. Levin, Starbursts near supermassive black holes: young stars in the galactic center, and gravitational waves in LISA band, MNRAS 374515 (2007) [astro-ph/ 0603583 ].

[30] M.C. Miller, Probing General Relativity with mergers of supermassive and intermediate-mass black holes, ApJ 618426 (2004) [astro-ph / 0409331 ]; S. Portegies Zwart et al, The ecology of star clusters and intermediate-mass black holes in the galactic bulge, ApJ 641319 (2006) [astro-ph/0511397].

[31] L.J. Rubbo, K. Holley-Bockelmann, and L.S. Finn, Event rate for extreme mass ratio burst signals in the laser interferometer space antenna band, ApJ 649 L25 (2006); C. Hopman, M. Freitag, and S.L. Larson, Gravitational wave bursts from the galactic massive black hole, MNRAS 378129 (2007) [astro-ph/0612337]; N. Yunes, C.F. Sopuerta, L. Rubbo, and K. Holley-Bockelmann, Relativistic effects in extreme mass ratio gravitational wave bursts, ApJ to appear (2008) [arXiv:0704.2612v1].

[32] Y. Mino, M. Sasaki, and T. Tanaka, Gravitational radiation reaction to a particle motion, Phys. Rev. D 553457 (1997) [gr-qc/9606018]; T.C. Quinn and R.M. Wald, An axiomatic approach to electromagnetic and gravitational radiation reaction of particles in curved spacetime, Phys. Rev. D 563381 (1997) [gr-qc/9610053]. 
[33] Y. Mino, Perturbative approach to an orbital evolution around a supermassive black hole, Phys. Rev. D 67084027 (2003) [gr-qc/ 0302075 ]; S.A. Hughes, S. Drasco, É.É. Flanagan, and J. Franklin, Gravitational radiation reaction and inspiral waveforms in the adiabatic limit, Phys. Rev. Lett. 94 221101 (2005) [gr-qc/ 0504 015]; N. Sago, T. Tanaka, W. Hikida, K. Ganz, and H. Nakano, The adiabatic evolution of orbital parameters in the Kerr spacetime, Prog. Theor. Phys. 115873 (2006) [gr-qc/0511151].

[34] S. Babak et al, 'Kludge' gravitational waveforms for a test-body orbiting a Kerr black hole, Phys. Rev. D 75024005 (2007) [gr-qc/ 0607007 ].

[35] A. Pound and E. Poisson, Osculating orbits in Schwarzschild spacetime, with an application to extreme mass-ratio inspirals [arXiv:0708.3033]; Multi-scale analysis of the electromagnetic self-force in a weak gravitational field, [arXiv:0708.3037]; Y. Mino, Modulation of the gravitational waveform by the effect of radiation reaction [arXiv:0711.3007].

[36] E. Poisson, The motion of point particles in curved spacetime, Living Rev. Rel. 76 (2004) [gr-qc/ 0306052 ]; C.O. Lousto, editor, Gravitational radiation from binary black holes: Advances in the perturbative approach, Class. Quant. Grav. 22 (2005).

[37] C.F. Sopuerta, P. Sun, P. Laguna, and J. Xu, A toy model for testing finite element methods to simulate extreme-mass-ratio binary systems, Class. Quant. Grav. 23251 (2006) [gr-qc/ 0507112 ].

[38] C.F. Sopuerta, P. Laguna, A finite element computation of the gravitational radiation emitted by a point-like object orbiting a non-rotating Black Hole, Phys. Rev. D 73044028 (2006) [gr-qc/0512028]. 